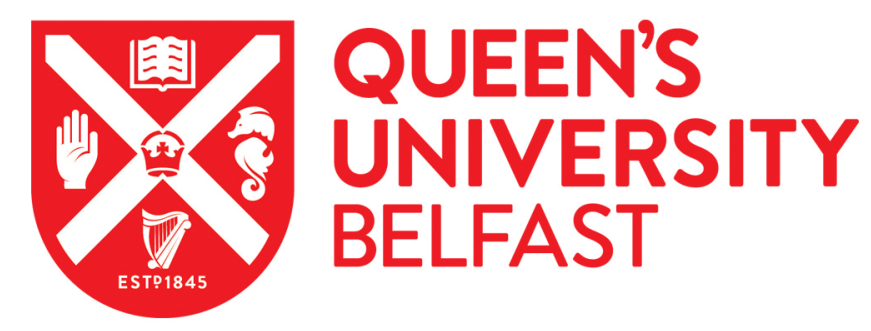

\title{
A Novel Edge Security Gateway for End-to-End Protection in Industrial Internet of Things
}

Khan, R., McLaughlin, K., Kang, B., Laverty, D., \& Sezer, S. (2021). A Novel Edge Security Gateway for End-toEnd Protection in Industrial Internet of Things. In 2021 IEEE Power and Energy Society General Meeting (PESGM 2021): Proceedings (IEEE Power \& Energy Society General Meeting (PESGM): Proceedings). Institute of Electrical and Electronics Engineers Inc.. https://doi.org/10.1109/PESGM40551.2019.8973954

Published in:

2021 IEEE Power and Energy Society General Meeting (PESGM 2021): Proceedings

\section{Document Version:}

Peer reviewed version

Queen's University Belfast - Research Portal:

Link to publication record in Queen's University Belfast Research Portal

\section{Publisher rights}

(c) 2021 IEEE.

This work is made available online in accordance with the publisher's policies. Please refer to any applicable terms of use of the publisher.

\section{General rights}

Copyright for the publications made accessible via the Queen's University Belfast Research Portal is retained by the author(s) and / or other copyright owners and it is a condition of accessing these publications that users recognise and abide by the legal requirements associated with these rights.

Take down policy

The Research Portal is Queen's institutional repository that provides access to Queen's research output. Every effort has been made to ensure that content in the Research Portal does not infringe any person's rights, or applicable UK laws. If you discover content in the Research Portal that you believe breaches copyright or violates any law, please contact openaccess@qub.ac.uk. 


\title{
A Novel Edge Security Gateway for End-to-End Protection in Industrial Internet of Things
}

\author{
Rafiullah Khan*, Kieran McLaughlin*, BooJoong Kang ${ }^{\dagger}$, David Laverty* and Sakir Sezer* \\ *Queen's University Belfast, Belfast, United Kingdom \\ $\dagger$ University of Southampton, Southampton, United Kingdom \\ Email: * ${ }^{*}$ rafiullah.khan, kieran.mclaughlin, david.laverty, s.sezer\}@qub.ac.uk, ${ }^{\dagger}$ b.kang@ @oton.ac.uk
}

\begin{abstract}
Many critical industrial control systems integrate a mixture of state-of-the-art and legacy equipment. Legacy installations lack advanced, and often even basic security features, risking entire system security. Existing research primarily focuses on the development of secure protocols for emerging devices or protocol translation proxies for legacy equipment. However, a robust security framework not only needs encryption but also mechanisms to prevent reconnaissance and unauthorized access to industrial devices. This paper proposes a novel Edge Security Gateway (ESG) that provides both, communication and endpoint security. The ESG is based on double ratchet algorithm and encrypts every message with a different key. It manages the ongoing renewal of short-lived session keys and provides localized firewall protection to individual devices. The ESG is easily customizable for a wide range of industrial application. As a use case, this paper presents the design and validation for synchrophasor technology in smart grid. The ESG effectiveness is practically validated in detecting reconnaissance, manipulation, replay, and command injection attacks due to its perfect forward and backward secrecy properties.
\end{abstract}

\section{INTRODUCTION}

Industrial Control Systems (ICS) tightly integrate physical processes and communication technologies. Unlike consumer IT devices, industrial systems are expensive with high installation complexity and a typical lifespan of several decades. Due to economic constraints, legacy devices are not feasible to replace and must co-exist with the latest devices in the continuously modernizing industry landscape. They often lack security features and rarely receive security patches/updates. Further, legacy protocols are plagued by cyber vulnerabilities, putting entire system security at stake [1].

Specifically, power companies have deployed a large number of legacy devices over the last two decades which have inherent security vulnerabilities. For example, most currently deployed phasor devices (i.e., Phasor Measurement Units (PMUs) and Phasor Data Concentrators (PDCs)) use the IEEE C37.118 protocol which is vulnerable to cyber-attacks [2]. The root cause of these attacks is the lack of end-point access control features and insecure communication protocols. Thus, a robust security framework needs to be developed to ensure safe and reliable synchrophasor operations.

\section{A. Related Work}

Several researchers have demonstrated cyber-attacks by exploiting vulnerabilities of legacy devices in power systems [2]-[4]. Different security technologies are generally adopted to defend ICS networks such as Intrusion Detection System (IDS) [5] and Intrusion Prevention System (IPS) [6]. These solutions generally raise a large number of false positives and false negatives due to the unpredictable nature of ICS dynamics e.g., power systems. Mistakenly dropping a critically important packet could be disastrous. Further, these solutions do not address fundamental communication security issues.

Prominent organizations have established working groups to develop future secure protocols for industrial devices. For example, a secure IEC 61850-90-5 protocol for PMU devices [7] and a secure cross-platform protocol for M2M communication (OPC UA) have been developed. However, emerging protocols can be adopted in new devices but security issues related to legacy devices still need to be addressed.

To bridge the technological gap between latest and legacy devices, authors in [8] proposed a protocol translation gateway. The gateway is attached to the industrial end-point that converts the legacy protocol into a secure modern protocol. However, all devices share the same encryption key using a group key management protocol. This could risk the entire system security if a single group device gets compromised. Authors in [9] proposed middleware that runs on both, the ICS device and the control center application to achieve mutual authentication and encryption. However, it uses static encryption and lacks end-point device protection.

\section{B. Paper Motivation and Contributions}

In general, the current research trend focuses on the development of secure protocols for new devices [7] and protocol translation proxies [8], [9] for legacy devices to address the communication security issues. These existing solutions normally use a single encryption key for each session or utilize the same key for all group members in the group key management framework [8]. This can put the entire system security at stake if an encryption key is compromised. Further, existing works do not protect ICS devices from network reconnaissance attacks (e.g., port scanning, ARP poisoning, etc).

The underpinning motivation of this paper is to design and develop a novel Edge Security Gateway (ESG) for end-toend protection in industrial systems. The ESG is a major step beyond existing ICS security gateway solutions by overcoming the above limitations. The key distinguishing features of ESG include: (i) a localized and customizable firewall for individual ICS devices, (ii) encryption of every message with a different 


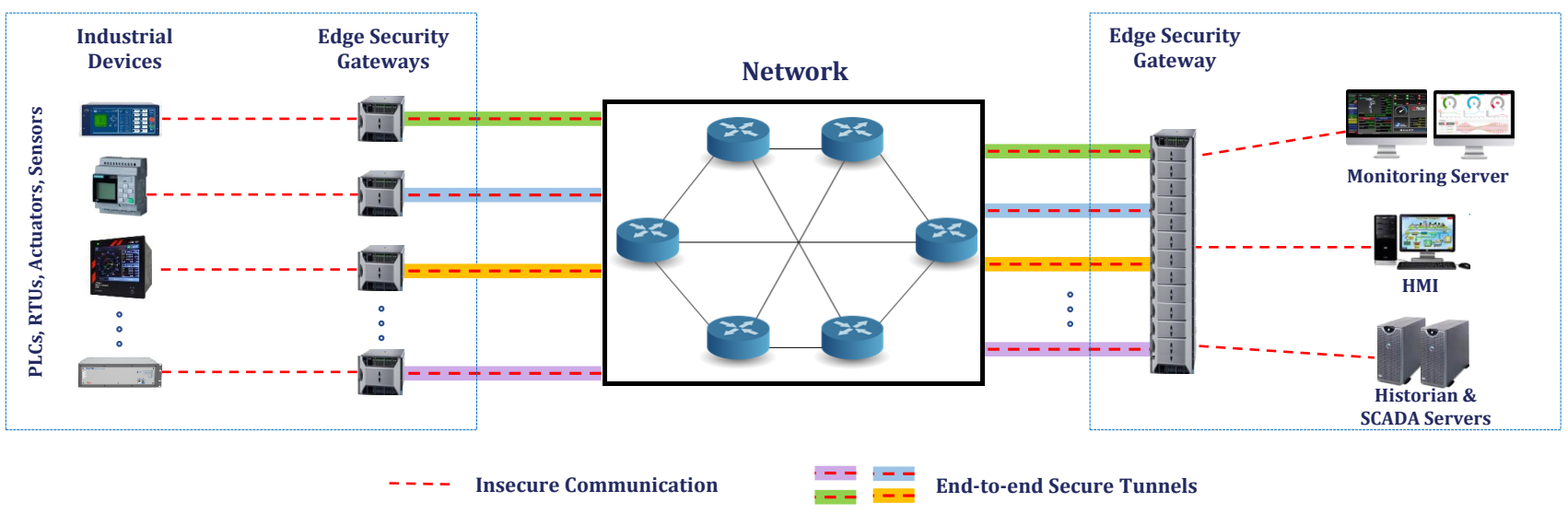

Figure 1. Overview of the proposed ESG concept. Each device has a secure tunnel with the control center using security credentials different from other field devices. Security credentials are dynamic and every message is encrypted with a different key. Each ESG manages its security credentials independently.

short-lived key, (iii) independent key management by every device, and (iv) forward and backward secrecy properties (i.e., past and future communications are still secure even if a current key gets compromised). These properties protect the ICS devices against network reconnaissance, Man-In-TheMiddle (MITM), replay, and command injection attacks.

This paper presents technical specifications for the practical implementation of the ESG. It investigates the necessary features and key architectural blocks for ensuring safe and robust industrial operations. As a use case scenario, the proposed ESG is implemented and functionally validated for synchrophasor technology in the smart grid. The testbed consists of a real microgrid facility in the laboratory. This paper considers different attack scenarios on the microgrid which could severely impact real-time control operations. It practically demonstrates that the ESG is effective in ensuring robust end-to-end security against network-based attacks.

\section{Proposed Edge Security Gateway}

Many industrial systems (e.g., power systems, oil and gas, etc) are geographically distributed which are managed via a local HMI or centrally controlled via remote access. History has shown that most successful cyber-attacks against ICS involve an internal system compromised at the first stage [10]. The attack then propagates to the rest of the network through reconnaissance (e.g., network queries, port scanning, ARP poisoning, etc). Since many industrial systems also consist of legacy equipment with no built-in security, reconnaissance becomes an easy task for the intruder after initial compromise of an internal machine. Thus, this paper proposes a novel ESG to protect the industrial systems. It ensures that all ICS communication is end-to-end encrypted with security credential managed individually by every device. By blocking network scanning, reconnaissance cannot be successful by an intruder even if an internal machine is compromised.

\section{A. ESG Concept}

A typical industrial system consists of control and monitoring applications, historian, SCADA servers, HMI, and several field devices (PLCs, RTUs, actuators, sensors, etc) as shown in Fig. 1. To secure the system, a low-cost ESG device is attached next to each field device. At the control center, a single ESG (one for all servers) or multiple ESG (one for each server) devices can be connected. The ESG has two Ethernet ports, one goes to the field device and the other goes to the network. It sits in the middle and blocks direct access to the ICS device based on the access control policies configured on it. Each ESG establishes an end-to-end encrypted tunnel with its remote peer's ESG. The ESG ensures that all network traffic is securely routed through the tunnel between the ICS device and servers in the control center (deployed locally or in the cloud). This makes ESG particularly useful for legacy devices with insecure protocols.

As depicted in Fig. 1, the ESG for each ICS device has its own encrypted tunnel with the remote peer. Security credentials are different for each tunnel and managed individually by each ESG device. This makes the system secure even if the security credentials of a specific tunnel are compromised (e.g., through physical access to an ESG by malicious intruders). Due to the independent management of security credentials by individual ESG devices, a single device compromise cannot impact the rest of the network. Further, security credentials are short-lived and a different encryption key is used for every message. Thus, the past and future communications remain secure even if a current key gets compromised by an attacker through cryptanalysis.

\section{B. ICS End Point Protection}

Unlike consumer devices (such as PCs), ICS devices do not have a complex OS with local access control and firewall policies configuration. The ESG solves this problem by providing localized security for each ICS device as shown in Fig. 2. It hides the ICS device behind and restricts its visibility in the network. The ESG allows connection to the ICS device only from the intended remote peer's ESG. It is configured to block reconnaissance related network queries and make ICS device non-discoverable to any malicious/compromised machine in 


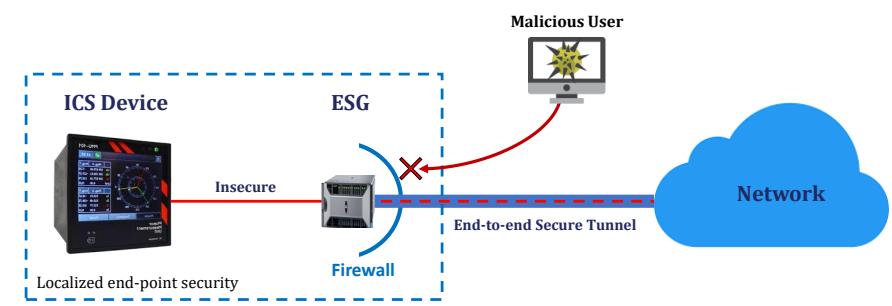

Figure 2. The ESG provides localized end-point security to each ICS device.

the local network. Reconnaissance is the first major step in the cyber kill chain process. By blocking reconnaissance, the ESG significantly reduces the possibility of cyber-attack against ICS devices.

\section{Dynamic Security based on Double Ratchet Algorithm}

The ESG uniquely encrypts every message with a different encryption key. The key management mechanism is based on the double ratchet algorithm. The double ratchet algorithm was initially developed in 2013 and became increasing popular over time due to its adoption in WhatsApp (i.e., signal protocol), Viber, Facebook Messenger, Google Allo, Skype and so on. Its foundation is based on the Diffie Hellman (DH) key exchange.

1) Diffie Hellman Key Exchange: The DH key exchange mechanism is depicted in Fig. 3. It can be observed that both the ESGs have no advanced knowledge of the shared secret key and derive it together. They both agree on a prime $\mathrm{p}$, and generator g. Both ESGs keep their private key secret and use a specific mathematical model to generate public key from it. They exchange their public keys with one another over the network and use a new mathematical equation to derive a common shared secret key. It can be observed in Fig. 3 that common shared secret key can only be derived if $\mathrm{p}$, g, private key, and remote peer's public key are known. Only the public key is shared over the network. Thus, intruders will not be able to derive the shared secret key even if they sniff network traffic in the middle (i.e., public keys alone are not enough to derive the shared secret key).

\begin{tabular}{|c|c|c|}
\hline ESG 1 & & ESG 2 \\
\hline$p, g$ & Step 1: Agree on prime and generator & $p, g$ \\
\hline a & Step 2: Generate a private key & $b$ \\
\hline$A=g^{a} \bmod p$ & Step 3: Calculate public key & $B=g^{b} \bmod p$ \\
\hline & & \\
\hline$=4$ & Step 4:Exchange public keys & $\widetilde{\sim n}$ \\
\hline $\begin{array}{c}K=B^{\mathrm{a}} \bmod \mathrm{p} \\
\mathrm{K}=\left(\mathrm{g}^{\mathrm{b}} \bmod \mathrm{p}\right)^{\mathrm{a}} \bmod \mathrm{p} \\
\mathrm{K}=\mathrm{g}^{\mathrm{ab}} \bmod \mathrm{p}\end{array}$ & Step 5: Calculate shared secret key & $\begin{array}{c}K=A^{b} \bmod p \\
K=\left(g^{a} \bmod p\right)^{b} \bmod p \\
K=g^{a b} \bmod p\end{array}$ \\
\hline 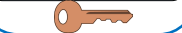 & & $0 \stackrel{2}{2}$ \\
\hline
\end{tabular}

Figure 3. Diffie Hellman key exchange mechanism used by the ESG.

2) Diffie Hellman Ratchet: The DH ratchet is based on the DH key exchange as depicted in Fig. 4. The process is as follows: (1) ESG-2 shares its public key with ESG-1 in the

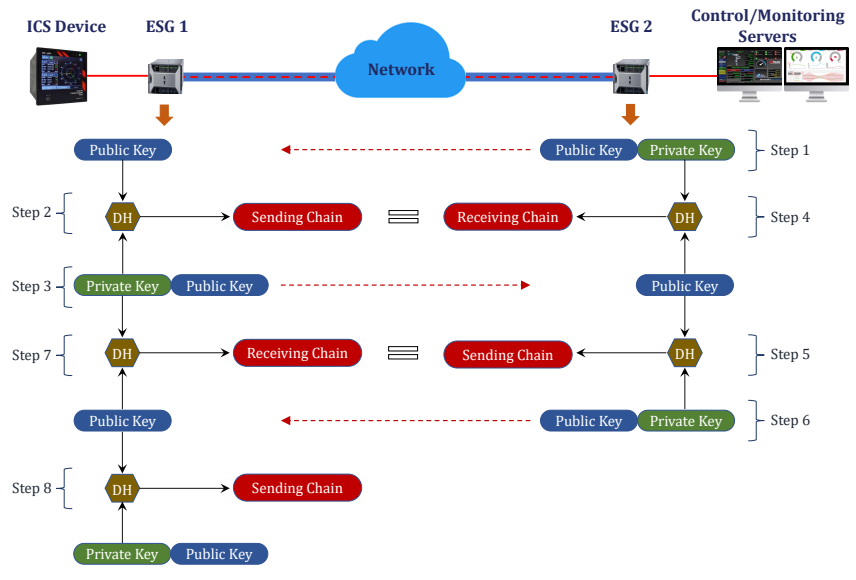

Figure 4. Diffie Hellman ratchet for ongoing renewal of secret key.

first message, (2) ESG-1 uses its own private key and ESG2's public key to derive secret key (i.e., Sending Chain), (3) ESG-1 encrypts the message with secret key and sends it to ESG-2 including its public key in the header, (4) ESG-2 uses its own private key and ESG-1's public key to derive secret key (Receiving Chain) and decrypts the received message, (5) ESG-2 randomly creates a new public-private key pair and uses new private key and ESG-1's public key to derive new secret key (Sending Chain), (6) ESG-2 encrypts new message with new secret key and sends it to ESG-1 including its new public key in the header, (7) ESG-1 uses its own private key and ESG1's new public key to derive secret key (Receiving Chain) and decrypts the received message, (8) ESG-1 randomly creates a new public-private key pair and uses new private key and ESG-2's public key to derive new secret key (Sending Chain), and so on. Thus, every message is encrypted with a different secret key and contains the sender's latest public key in the header.

3) Double Ratchet Mechanism in the ESG: The ESG uses double ratchet for enhanced security and perfect forward secrecy. As depicted in Fig. 5. It combines DH ratchet with the Key Derivation Function (KDF) of the symmetric key ratchet. The KDF is a cryptographic function that converts secret root key and input DH data into a new root key that is indistinguishable from random. As shown in Fig. 5, a KDF chain is formed in which output of a KDF is used as the root key for the next KDF. The use of KDF chain improves resilience (KDF output key cannot be derived by the adversary as input root key is unknown), forward secrecy (KDF chain output keys generated before the compromise cannot be derived by the adversary), and break-in recovery (KDF chain output keys generated after the compromise cannot be derived by the adversary).

The sending and receiving chain keys are used by the send ratchet and receive ratchet, respectively (as shown in Fig. 6). As highlighted in Fig. 6, the ratchets can rotate in only one direction i.e., a new key is generated by taking the previous key as input but vice versa is not possible. Each ESG is managing the DH ratchet that then updates the send and receive ratchets. For sending a message, ESG applies symmetric key ratchet 


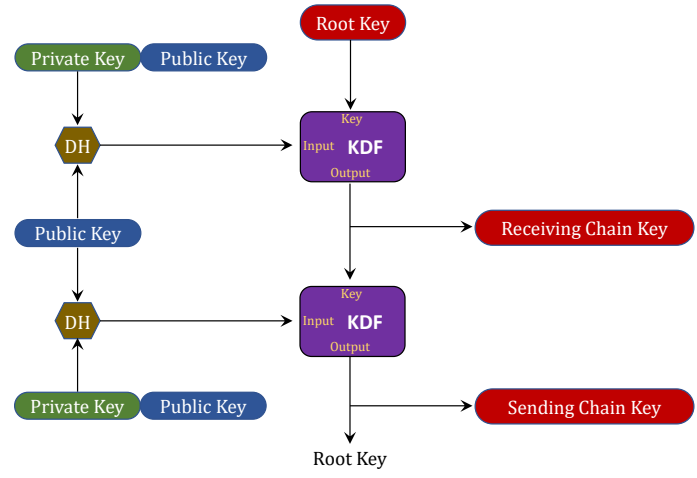

Figure 5. Double ratchet mechanism. DH ratchet output is used as input for the KDF to generate sending and receiving chain keys.

on the sending chain key to generate a message key (used for encryption). To decrypt a received message, ESG applies symmetric key ratchet on the receiving chain key to generate a message key (used for decryption).

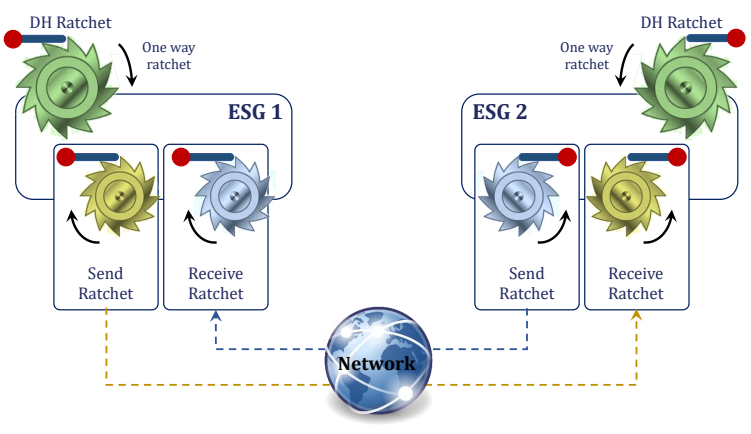

Figure 6. Double ratchet mechanism overview in the ESG.

\section{TEST-BED AND EXPERIMENTAL EVALUATION}

\section{A. Use Case: Synchrophasor Technology in Smart Grid}

This paper validates ESG functionalities for synchrophasor technology. Synchrophasor technology uses phasor devices (PMUs/PDCs) for Wide-Area Monitoring, Protection And Control (WAMPAC) in the smart grid. Most currently deployed phasor devices use the IEEE C37.118 protocol which is vulnerable to cyber-attacks. Thus, ESG can play a vital role in ensuring trustworthy WAMPAC operations.

\section{B. Experimental Testbed}

The testbed consists of a microgrid, a PMU, two ESG machines, control application, and an attacker as shown in Fig. 7. The microgrid is located in Belfast (UK) and is comprised

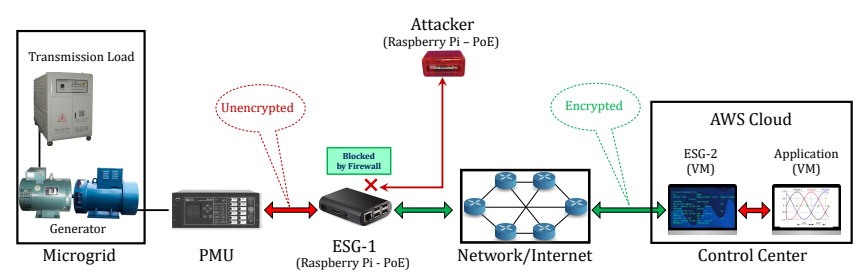

Figure 7. Testbed for experimental validation of ESG functionalities of an alternator and a 3-phase transmission load. The PMU measures electrical quantities from microgrid in real-time and sends them to the control application. The attacker and ESG1 software are hosted on Raspberry Pi devices with Power over Ethernet (PoE) support. ESG-2 and control application are hosted in virtual machines (VMs) on Amazon AWS cloud. London (UK) is selected as the location for AWS cloud VMs. The attacker software is capable of executing reconnaissance, stealthy MITM manipulations, command injection, and replay attacks against the PMU (details are provided in [2]). The ESG software is implemented in Linux OS using Python programming language and PyCrypto libraries. It uses AES256-GCM algorithm for encryption and HMAC-SHA-256 algorithm for cryptographic signature. The pictorial view of the testbed components is shown in Fig. 8.

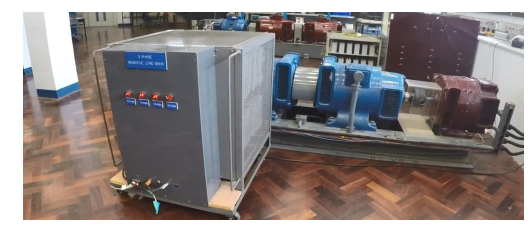

(a) Microgrid with 3-Phase Transmission Load

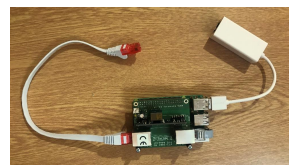

(c) ESG

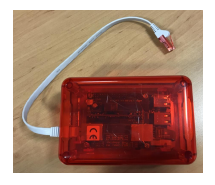

(d) Attacker

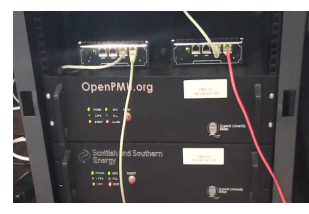

(b) PMU

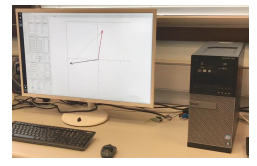

(e) PMU Controller
Figure 8. Pictorial view of the testbed components.

\section{Functional Validation of the ESG}

The ESG functionalities were successfully validated for different attack scenarios. Without ESG devices in Fig. 7, the attacker was able to successfully scan the network and discover the PMU. After connecting ESG devices, the attacker was unable to discover the PMU. This is due to the fact that the ESG creates a control zone between its two interfaces and selectively forwards packets between them. The configured policies block network scanning traffic and protect the PMU from reconnaissance attack.

Reconnaissance is the first major step in most cyberattacks. By blocking reconnaissance, the ESG automatically prevents MITM manipulations, replay, and command injection attacks. However, for the sake of experiments, now ESG-1 was configured to forward network scanning traffic between its two interfaces. This enabled the attacker to discover the PMU device. Since the packets were encrypted, the attacker was unable to modify the packets according to the IEEE C37.118 protocol. The random modifications to packet content by attacker were successfully detected by the ESG due to the violation of the cryptographic signature. For replay attack, the attacker stored a copy of the packet and forwarded the outdated packet to the PMU device. The ESG successfully detected it as the replayed packet was encrypted using an old encryption key. Now, the attacker was configured to inject non-encrypted IEEE C37.118 command message to the PMU. 


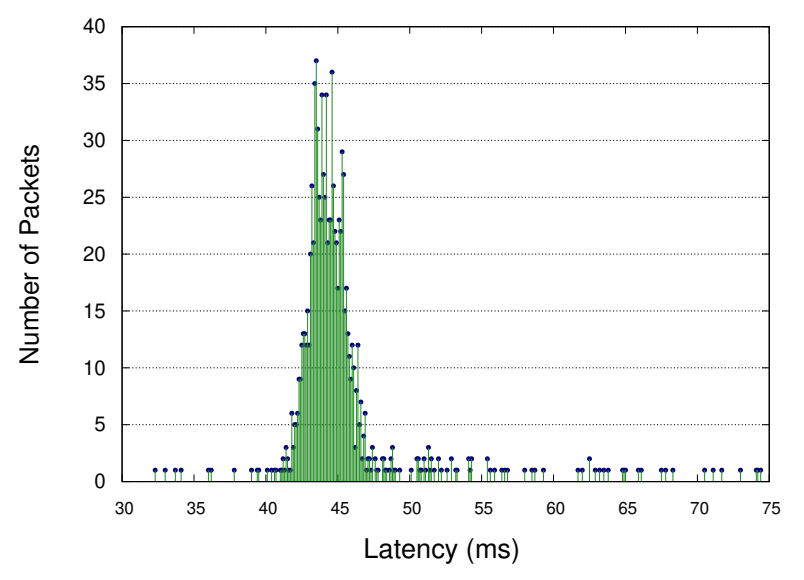

Figure 9. Distribution of latency values (rounded to one decimal place).

The ESG automatically detected it and ignored its processing. This is due to the fact that the ESG works as a proxy and tries to decrypt every message received. TCP session and double ratchet mechanism reset if a message is lost and ESG automatically performs initialization again. Such event is also reported to operator in logs.

\section{Communication Latencies}

Latencies can severely affect the real-time industrial operations. The microgrid testbed under consideration has a $100 \mathrm{~ms}$ limit for communication latencies. In the cloud-based system (shown in Fig. 7), several factors can influence the communication latency including the ISP, access technology and the ESG device processing power. Fig. 9 depicts the distribution of communication latencies for 1000 packets when ESG devices are connected. It can be observed that majority of the packets have one-way communication latency within the range of 40$50 \mathrm{~ms}$. Table I presents latencies before and after connecting the ESG devices. It can be observed that incremental latencies with ESG are much smaller than $100 \mathrm{~ms}$ requirement due to sufficient computing power of Raspberry Pi and advancement in Internet access technologies. This validates that a low-cost ARM-based device has enough computing power to host ESG software for time-critical synchrophasor technology.

Table I

COMMUNICATION LATENCIES AVERAGED OVER 1000 TRIALS.

\begin{tabular}{lcccc}
\hline & \multicolumn{4}{c}{ Latencies (ms) } \\
\cline { 2 - 5 } & Min & Avg & Max & Std. Dev \\
\hline Without ESG & 9.132 & 10.823 & 39.396 & 3.919 \\
With ESG & 32.265 & 45.131 & 74.361 & 4.195 \\
\hline
\end{tabular}

\section{CONCLUSions}

Industrial systems usually consist of a mixture of stateof-the-art and legacy devices. Legacy devices are normally vulnerable to cyber-attacks, risking the entire system security. To address security challenges, the current research trend primarily focuses on investigating future secure ICS protocols for new devices [7] or developing protocol translation features for legacy devices [8], [9]. However, they lack the end-point protection features and normally rely on a single key for encryption.

In contrast to previous works, the ESG inherits several unique properties essential for robust industrial system security. The ESG ensures that every message is encrypted with a different short-lived key. A security key compromise could be disastrous for industrial systems if used by an attacker with malicious intends. The ESG overcomes this limitation by incorporating perfect forward and backward secrecy properties in its security mechanism. This means that past and future communications are still protected even in case of a security key compromise. Additionally, every ESG device is managing its key independently. Thus, a compromise of one device cannot impact the entire network.

For validation of the ESG concept, this paper presented the design and deployment scenarios for synchrophasor technology in the smart grid. The ESG successfully ensured endto-end communication security in the synchrophasor-based testbed which is crucial for trustworthy WAMPAC operations. The ESG was effective in preventing reconnaissance, MITM manipulation, replay, and command injection attacks. By isolating the ICS end-point device from the network and creating a virtual buffer zone, the ESG successfully blocked network and port scanning which are fundamental in the cyber kill chain process. It is experimentally validated that a low-cost ARM-based ESG has enough computing power to meet latency requirements for synchrophasor technology. The ESG can be easily customized for other industrial sectors and hosted on a more powerful machine to meet their latency requirements.

\section{REFERENCES}

[1] T. Alladi, V. Chamola, and S. Zeadally, "Industrial Control Systems: Cyberattack trends and countermeasures," in Computer Communications, Vol 155, pp 1-8, 2020.

[2] R. Khan, K. McLaughlin, J. H. D. Laverty, H. David, and S. Sezer, "Demonstrating cyber-physical attacks and defense for synchrophasor technology in smart grid," in 16th IEEE PST, 2018.

[3] C. Tu, X. He, X. Liu, and P. Li, "Cyber-attacks in pmu-based power network and countermeasures," IEEE Access, 2018.

[4] J. Graham, J. Hieb, and J. Naber, "Improving Cybersecurity for Industrial Control Systems," in Int. Symp. on Industrial Electronics, 2016.

[5] A. Chavez, C. Lai, N. Jacobs, S. Hossain-McKenzie, C. B. Jones, J. Johnson, and A. Summers, "Hybrid intrusion detection system design for distributed energy resource systems," in IEEE CyberPELS, 2019.

[6] J. Brugman, M. Khan, S. Kasera, and M. Parvania, "Cloud based intrusion detection and prevention system for industrial control systems using software defined networking," in Resilience Week (RWS), 2019.

[7] "Communication networks and systems for power utility automation - part 90-5: Use of iec 61850 to transmit synchrophasor information according to ieee c37.118," in IEC Technical Report.

[8] R. Khan, K. McLaughlin, J. Hastings, D. Laverty, and S. Sezer, "Intertechnology bridging gateway: A low cost legacy adaptation approach to secure industrial systems," in IEEE PES-GM, 2018.

[9] T. A. Rizzetti et al., "Cyber security and communications network on scada systems in the context of smart grids," in UPEC, 2015.

[10] R. Khan, K. McLaughlin, D. Laverty, and S. Sezer, "Threat Analysis of BlackEnergy Malware for Synchrophasor based Real-time Control and Monitoring in Smart Grid," in 4th ICS-CSR, August 2016. 